\section{Take a breath of polarized}

\section{noble gas}

Ernst W. Otten,

Institut für Physik, Johannes Gutenberg-Universität Mainz

$T$ sn't that title just a piece of nonsense designed to catch your 1 attention? Why should you breathe in the inert noble gas knowing that you need oxygen instead? Maybe an aging singer could mix some helium to his breath when his voice does not meet the high notes anymore. Of course, divers exchange nitrogen with helium since even under pressure it hardly dissolves in the blood and hence averts the danger of bubbling and embolism when the pressure is released. But do not mix it up with a xenon bottle! You would quickly lose your consciousness, since xenon penetrates into the blood and further into the brain with anaesthetizing effect. The real trick, however, is breathing spin-polarized noble gas! Why?

Almost a decade ago, a Princeton-StonyBrook collaboration published a seminal paper in NATURE [1] in which they showed for the first time a magnetic resonance image (MRI) of excised mouse lungs filled with spin-polarized ${ }^{129} \mathrm{Xe}$ (Fig. 1 left). The image was not formed as usual from the MRI signal of the protons in the tissue but from the pre-polarized xenon nuclei serving as a contrast agent, so to say. The decisive novelty in MRI was the use of spins which had been pre-polarized beforehand to a so-called hyperpolarization far beyond thermal equilibrium. In contrast the usual MRI signal derives from the Boltzmann polarization $P_{\mathrm{B}}$ of nuclear spins, which is established in the magnetic field $B_{0}$ of the tomograph within a so-called longitudinal relaxation time $T_{1}$ of order $1 \mathrm{~s}$ to the thermal equilibrium value of

$$
P_{B}=\frac{n^{+}-n^{-}}{n^{+}+n^{-}}=\tanh \left(\frac{\mu_{I} B_{0}}{k T}\right)
$$

where $n^{+}$and $n$ denote the number of spins oriented parallel and anti-parallel to $B_{0}$, respectively. In a typical tomograph field of $B_{0}=1.5 \mathrm{~T}$ the protons at body temperature reach a polarization of only $P_{\mathrm{B}} \approx 510^{-6}$. Therefore, MRI is, first of all, a fight for signal to noise ratio. This is virtually lost if, in addition to the low $P_{\mathrm{B}}$, the spin density is lowered from condensed matter level to that of a gaseous medium. Hence, the air filled spaces of the lungs, for instance, could not be imaged by NMR methods. Indeed the lung tissue itself hardly yields any signal since it is very porous and lacking in protons (Fig. 1 right).

\section{Optical pumping}

Only a radical increase in the spin polarization to far beyond $P_{\mathrm{B}}$ can help us to perform MRI on a gaseous medium. Polarizing beyond $P_{\mathrm{B}}$ succeeds with so-called dynamical methods. In essence, they transfer polarization from one system to the other, for instance from electrons to protons as in the case of the well known polarized proton targets. A particularly elegant solution well suited to gases is Alfred Kastler's method of optical pumping (OP) which won him the Nobel Prize in 1966. It transfers polarization from photons to atoms by resonant absorption of circularly polarized light. Grosso modo, the atomic system gains $1 \hbar$ of angular momentum per absorbed photon. Not a bad bargain! Bowever, things do not work that easily. Only a few atomic species are well suited to optical pumping, namely alkalis and helium. Molecules are excluded since they lose their polarization in gas kinetic collisions, as do atoms in other than spherical S-states. Moreover, an atomic vapour is already becoming optically thick for a strong resonance line at a column density of order $10^{10}$ atoms $/ \mathrm{cm}^{2}$. At a normal density of $3 \cdot 10^{19} / \mathrm{cm}^{3}$, the light would neither get in nor out. This explains why it is only for thin vapours that OP is a well established and widely used art, culminating nowadays in the spectacular experiments involving the cooling and manipulating of atoms by lasers. Polarizing large quantities of gas by OP is in a different category, however. It only succeeds by indirect means and only for noble gases. The light is absorbed by a thin medium whose polarization is then transferred to the dense noble gas by spin exchange mechanisms during gas kinetic collisions. Polarizing the whole ensemble takes up time, ranging from seconds to hours, during which the dense gas must not lose its polarization, either by gas kinetic or by wall collisions. Only noble gases can fulfil this demand with helium being better than xenon, strictly following the ranking of nobility. There are two methods in this game, already known since the early sixties. It is only in recent years that they have reached their quantitative goal after extensive R\&D work.

We begin with the so-called rubidium spin exchange method ( $R b S E$ ), discovered at Princeton in 1960 and pursued there ever since. Here, the unpaired spin of the valence electron of $\mathrm{Rb}$ is polarized by OP via the strong resonance line at $795 \mathrm{~nm}$ connecting the $5 s^{2} S_{1 / 2}$ ground state to the $5 \mathrm{p}^{2} \mathrm{P}_{1 / 2}$ excited states (left of Fig. 2). Rb electron spins then transfer their polarization through magnetic hyperfine coupling to the nuclear spins of a noble gas which is admixed at much higher density up to several bars. Due to the weakness of hyperfine coupling, this kind of spin exchange is a rare process occurring with a probability of order $10^{-4}$ for xenon and $10^{-7}$ for helium during a gas kinetic encounter of the partner atoms. As a result, it takes from minutes for ${ }^{129} \mathrm{Xe}$ up to many hours for ${ }^{3} \mathrm{He}$ before the noble gas attains its equilibrium nuclear polarization.

The other method can be traced back to the early sixties when a group from Rice University published the result of their experiment on metastable exchange optical pumping (MEOP) which exclusively applies to the species ${ }^{3} \mathrm{He}$. In MEOP metastable ${ }^{3} \mathrm{He}$ atoms are produced in the $1 s 2 s^{3} \mathrm{~S}_{1}$ state at a relative population of $10^{-6}$ in a low pressure plasma at about 1 mbar. These metastable atoms are then polarized by OP at $\lambda=1083 \mathrm{~nm}$ connecting to the $1 \mathrm{~s} 2 \mathrm{p}^{3} \mathrm{P}_{0}$ state (right of Fig. 2). With a powerful laser source it takes only a fraction of a microsecond to polarize this metastable state.

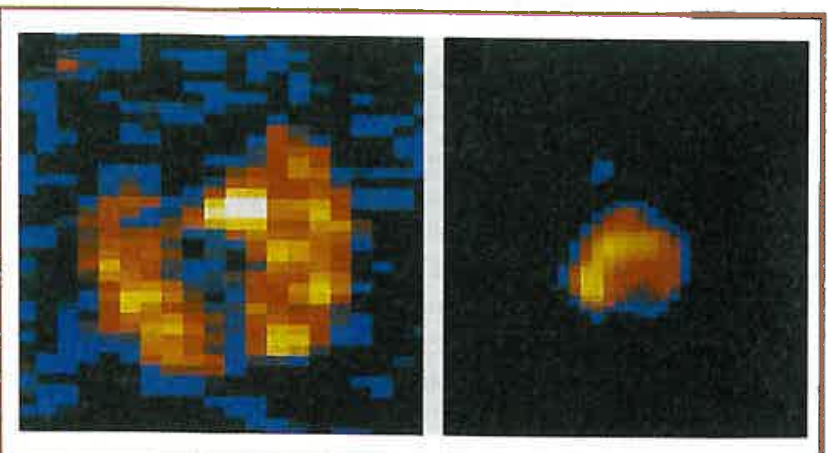

A Flo. \%: Left: The pioneering MRI of hyperpolarized ${ }^{129} \mathrm{Xe}$ injected into excised mouse lungs.

Right: Proton MRI of the same subject showing only the tissue of the heart in the centre, but no proton signal from the lungs [1]. 
Fortunately, this characteristic pumping time $\tau_{\mathrm{OP}}$ matches fairly well to the metastable exchange time $\sigma_{\text {ex }}$ within which the metastable excitation energy is transferred to the next atom, leaving the former in the atomic ground state with a polarized nucleus. The next atom is then ready for pumping, thus forming a fast catalytic chain of successive energy transfers and pumping action. Within a few seconds the ${ }^{3} \mathrm{He}$ plasma attains a nuclear polarization of about $50 \%$ and saturates in the range of $80 \%$. MEOP owes its rapidity to the very large metastable exchange cross section $\left(\sigma_{\mathrm{ex}} \approx 10^{-15} \mathrm{~cm}^{2}\right)$ which is driven by the dominant electrostatic rather than by the weak hyperfine interaction.

In Europe, Kastler's and Brossel's laboratory in Paris devoted its experimental and theoretical activity from the beginning in the fifties to the foundations of the physics of OP, including both indirect methods of noble gas pumping. In particular it has a strong tradition in studies of MEOP which lead to a complete theory of the pumping and exchange dynamics [2] and to the development of suitable laser sources.

\section{Polarizing and handling larger quantities of noble gas}

The motivation for polarizing ${ }^{3} \mathrm{He}$ in large quantities did not come from MRI but from particle and nuclear physics where there was a need for a polarized neutron target or at least a substitute for it. As such, polarized ${ }^{3} \mathrm{He}$ serves best since the spin and magnetic moment of the nucleus stem almost entirely from the single neutron. A SLAC-Princeton-collaboration realized a successful high pressure target polarized on-line by the RbSE method. It served to measure the polarized neutron structure function in deep inelastic electron scattering [3]. A Mainz-Paris-Collaboration realized the first dense MEOP target which led to the measurement of the electric form factor of the neutron in quasi-elastic electron scattering [4]. Another motivation was to realize a broadband spin filter for neutron beams, based on the huge spin dependent absorption cross section of ${ }^{3} \mathrm{He}$ [5] (see also W. Heil at the International Workshop HELION02, Oppenheim, Germany (2002), [6]). These experiments needed quantities of order 1 standard litre and a polarization of order 40 to $50 \%$. Readers should note that these numbers match quite well to what is needed for performing MRI of a human lung. That is why this technique could be applied so quickly once the idea was born. All the necessary R\&D work had been carried out beforehand for the purposes of fundamental physics research. This is yet another example proving that fundamental research is a most fruitful source of innovation.

High production rates at high polarization could only be achieved through the advent of powerful pumping lasers at the required infrared wave lengths. In particular, $\mathrm{RbSE}$ scales rather strictly with the laser power available. A low SE cross section requires high $\mathrm{Rb}$ density in order to reach satisfactory exchange rates for the spins of the noble gas. Nowadays, one works under conditions where the $\mathrm{Rb}$ vapour has an optical thickness of order of 1000 absorption lengths. Hence, it takes of order $100 \mathrm{~W}$ for the laser beam to break through this wall by saturated absorption. Arrays of diode lasers can now provide this power routinely at reasonable cost. At the same time any fluorescence light must be totally suppressed by quenching the $\mathrm{Rb}$ excitation by a high dosage of admixed nitrogen (Fig. 2a). Rb SE polarization units now yield routinely production rates of order 0.1 standard $\mathrm{l} / \mathrm{h}$, reaching degrees of polarization in the range of $30 \%$ to $50 \%$ at maximum (see also G. Cates in [6]). ${ }^{129} \mathrm{Xe}$ can be polarized at considerably larger yields but to a somewhat smaller degree, usually.

In MEOP the He plasma yields only an optically thin layer of metastables, hence the laser beam is used to irradiate a whole battery of long plasma tubes (Fig. 2f). At an intensity of about
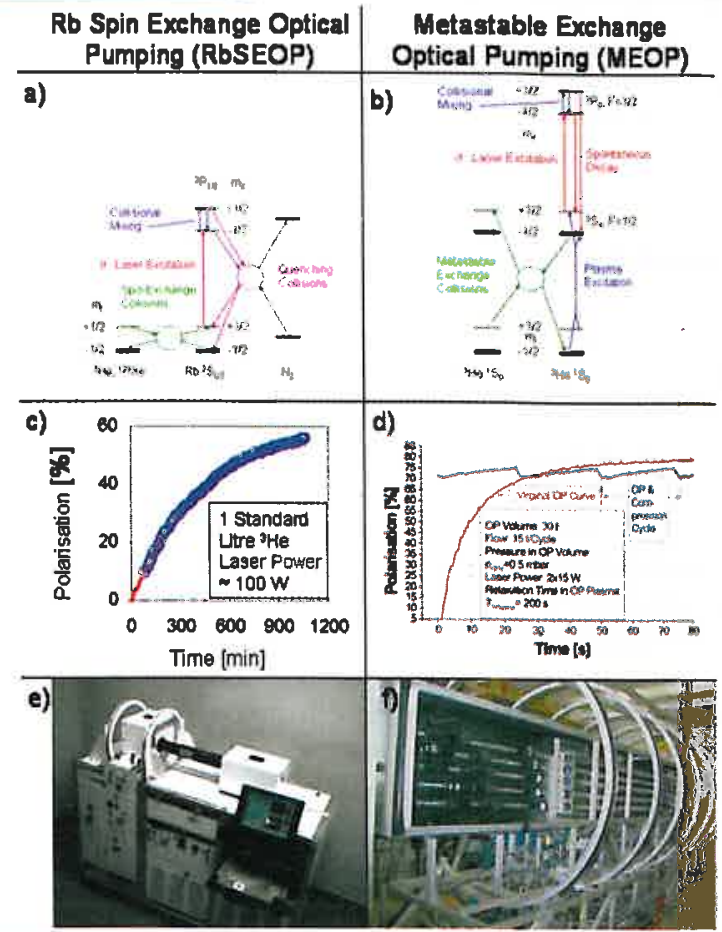

A Fig. 2: Polarizing noble gases by RbSEOP (left) and MEOP (right).a) and b) show the respective pumping schemes, c) and d) up-polarization curves for ${ }^{3} \mathrm{He}, \mathrm{e}$ ) and $\mathrm{f}$ ) the respective polarizers, namely the first commercial one (by Amersham Health) following RbSE and a MEOP laboratory setup at Mainz [5], now fabricated by IC-Automation.

$1 \mathrm{~W} / \mathrm{cm}^{2}$ the OP cycle is speeded up to its saturation limit of about $10^{-7} \mathrm{~s}$, the natural lifetime of the upper P-state. The scaled-up MEOP spin factory at Mainz yields about 5 standard $1 / \mathrm{h}$ at $P=50 \%$, rising beyond $70 \%$ at reduced flow (Fig. $2 \mathrm{~d}$ ). However, it is necessary to compress polarized ${ }^{3} \mathrm{He}$ from less than $1 \mathrm{mbar}$ in the OP plasma to at least atmospheric pressure at which it has to be inhaled, e.g. It took the Mainz group more than a decade of research to develop step by step a satisfactory solution such that no polarization is lost during compression (see also E. W. Otten [6]). Note that relaxation predominantly occurs during wall collisions, hence scales with the surface to volume ratio and that this ratio really explodes at large compression factors in a piston for example.

Today, both possible methods in the game have reached satisfactory physical and technical standards: RBSE with a compact set-up (Fig. 2c) for modest production rates fulfils the demands of a single research or clinical unit, MEOP with an elaborate apparatus for large-scale production capable of serving many users. The latter statement provokes the question of whether the polarization can survive shipping to a client. For ${ }^{3} \mathrm{He}$ the answer is "yes" but the "how" is not trivial! In the early days of MEOP, one had already found that polarized ${ }^{3} \mathrm{He}$ bottled in nonmagnetic vessels such as those made from glass or metals, relaxes quite slowly. Obviously, surface relaxation of a gas scales with adsorption time and energy. These are smallest for He leaving its spin least chance to flip in an encounter with a paramagnetic centre. Any knowledge beyond these qualitative statements is empirical, gained mostly by trial and error. Anyway, carefully cleaned and baked bulbs from special glass nowadays reach $T_{1}$ values of order $100 \mathrm{~h}$ for ${ }^{3} \mathrm{He}$, completely satisfactory for storage and shipment. To that end the bulb is 
housed in a $\mu$-metal box providing a small homogeneous holding field. Within the framework of an EU research project on polarized helium imaging of lungs (PHIL) [7], the delicate medium is now routinely delivered from the Mainz physics institute to scanners in clinics at Copenhagen, Mainz and Sheffield. The shorter relaxation time of ${ }^{129} \mathrm{Xe}$, on the other hand still requires on-site polarization.

\section{MR imaging with hyperpolarized gases}

Once the idea of using hyperpolarized gases for MRI was published [1] and sufficient quantities could be provided, its implementation into existing MRI techniques was realized very rapidly. The reason for this is readily found in the brilliant standards of signal processing, in the high flexibility, and in the wide diversification of this well established technology. Still, it is interesting for a physicist to know how it is done. It was said already that the basic capital of optically pumped gases lies in their extreme hyperpolarization which exceeds the Boltzmann value $P_{\mathrm{B}}$ (eq. 1) by five orders of magnitude and hence even overcompensates the loss in spin density as compared to protons in tissue. At $P=50 \%$ and diluted in the air of the lungs ${ }^{3} \mathrm{He}$ still offers a nuclear magnetization $\boldsymbol{M}$ much stronger than that stemming from protons in tissue. But once this hyperpolarization is used up by exciting magnetic resonance, it is gone forever, whereas $P_{\mathrm{B}}$ of the protons recovers within seconds and is then ready for the next NMR pulse. Usual MRI sequences need of order $100 \mathrm{MR}$ pulses at different field gradients to produce an image of decent resolution. Hence, one has to observe economy with the hyperpolarized spins and use only part of them for each MR pulse. Let us adjust for instance the MR pulse such that $M$ flips from its static orientation along $B_{0}$ by an angle of say $\vartheta=6^{\circ}$ towards the transverse plane, then we have already a transverse component of $M_{\perp}=|M| \sin 6^{\circ}=0.1 M$ precessing after the pulse at the Larmor frequency

$v_{\mathrm{L}}=\gamma B$

around $B$ and causing an induction signal in the antenna coil. It is already $10 \%$ of the maximum signal achieved with a $90^{\circ}$ flip. But the remaining longitudinal magnetization $M=|\boldsymbol{M}| \cos 6^{\circ}=0.995$ $M$ has dropped only by $0.5 \%$ and hence can easily feed another $100 \mathrm{MR}$ pulses as required. Summarizing, ${ }^{3} \mathrm{He} \mathrm{MRI}$ can count on a signal strength comparable to that of proton MRI.

Each MR pulse is followed by applying a series of field gradients $\nabla_{i} B$ over the sample which encode the position of its spins with a particular frequency shift

$$
\Delta v_{i}(r)=\gamma\left(\nabla_{i} B \cdot r\right)
$$

The antenna then receives an integral signal from the whole sample

$$
S_{i} \propto e^{i 2 \pi \gamma B_{0} t} \int_{\text {Sample }} M(r) e^{i 2 \pi \gamma\left(\nabla_{i} B \cdot r\right)} \mathrm{d} \tau
$$

that is a Fourier transform of the magnetization. The series of signals $S_{\mathrm{i}}$ can then be retransformed back into the sought for $M(r)$. That is all we need in principle. In reality there are many additional tricks in the game which are standard in modern MRI. Usually, one already applies a gradient during the MR pulse, say in the zdirection; this selects a certain slice in the xy-plane to be imaged. The thinner the slice the better the resolution, but at the cost of the

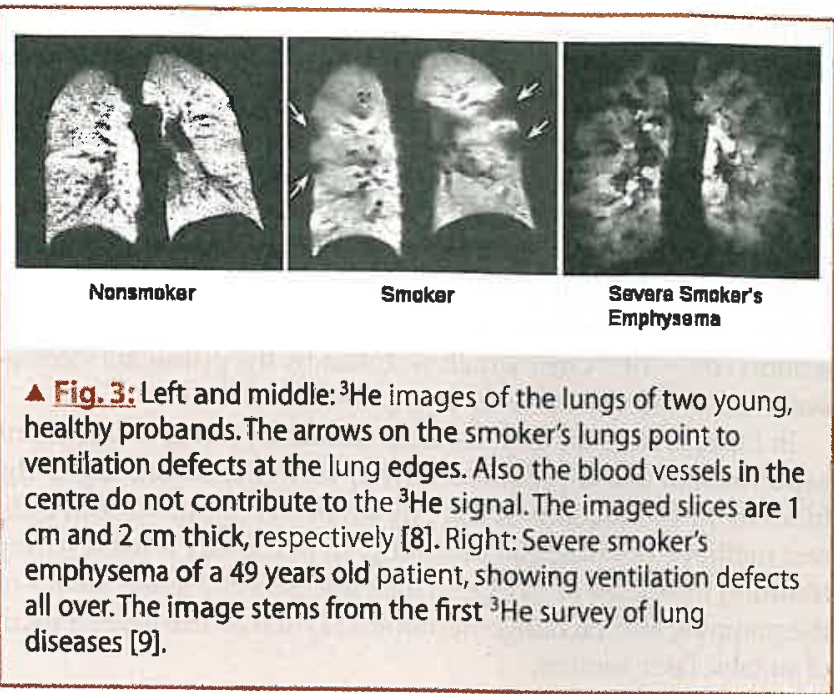

signal strength. During free precession one first applies a gradient, for example in the $\mathrm{x}$-direction and reverses its sign later waiting for the so-called spin echo at which the spins are coherently rephased. During the echo another field gradient is applied in the $\mathrm{y}$-direction. In this way one can extract from the time and frequency distribution of the echo the $\mathrm{x}$ - and $\mathrm{y}$-distribution of $M(r)$ in the slice simultaneously. Fig. 3 from the Mainz Clinics shows some ${ }^{3} \mathrm{He}-\mathrm{MRI}$ slices of human lungs with an in-plane resolution of $256 \cdot 256$ pixels $[8,9]$.

There are a few more features of hyperpolarized gas MRI which play specific roles. The gain in imaging time compared to ordinary MRI is of importance. It results from the fact that one does not need to wait for the recovery of the Boltzmann polarization (taking about $1 \mathrm{~s}$ ) in between consecutive MR pulses. This plays a role in lung imaging, since one cannot hold one's breath for much longer than $\approx 20 \mathrm{~s}$. Regarding short imaging times, ultrafast sequences have been developed which show a 3-dimensional movie of the breathing process with an apparent time resolution of $20 \mathrm{~ms}$. One may find such impressive movies on the website of the group at Charlottesville [10]; see also [11].

There is yet another reason not to stretch hyperpolarized MRI over much more than about $15 \mathrm{~s}$ - this is the relaxation time of ${ }^{3} \mathrm{He}$

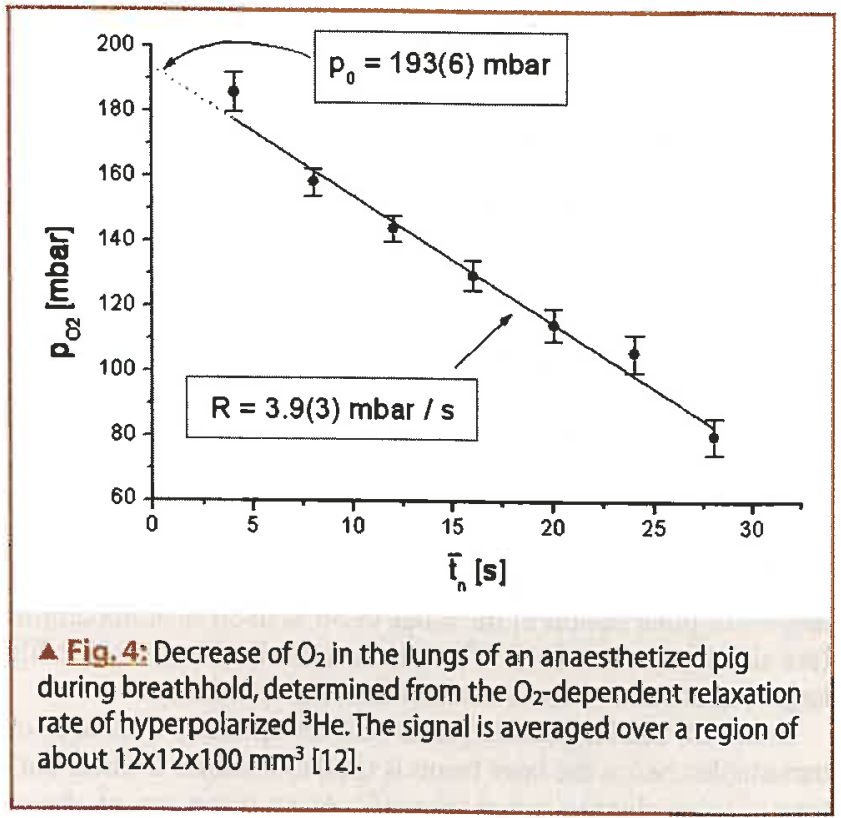


in the presence of the oxygen in the lungs. The reason for this relaxation effect is the strong dipolar coupling to the paramagnetic oxygen molecules. Hence, the hyperpolarization just fades away during the period when the breath is held. A physicist, however, is trained to look always at both sides of the coin. Here, he easily recognizes on the other side a chance to image the actual oxygen concentration in the lungs and moreover, how it drops during the holding of breath due to oxygen uptake which is the principal lung function. One does so by precisely measuring the time evolution of $P$ in consecutive images (Fig. 4) [12].

It is not only the longitudinal relaxation time $T_{1}$ that counts, since the transverse $T_{2}$ characterizes the decay of the coherence of the free Larmor precession and hence of the induction signal. In the field gradient of the tomograph this dephasing happens necessarily for the entire spin ensemble with a time $T_{2} \approx 1 / \Delta v$ according eq. 3. This is a reversible process as long as the spins do not move and the gradient is under control. This fact denotes the origin of Hahr's famous spin echo technique which has been known for 50 years. However, the coherence decays irreversibly when the atoms diffuse at random through the gradient. For ${ }^{3} \mathrm{He}$ the diffusion coefficient in air is about $0.8 \mathrm{~cm}^{2} / \mathrm{s}$, orders of magnitude larger than for protons in tissue or water. For this reason too, gas MRI has to be speeded up. The imaging gradient must not last longer than a couple of milliseconds. Again, let us have a look at the other side of the coin. The gradient sequence may be tailored such that the signal strength and hence the image are just weighted with the local diffusion coefficient (Fig. 5 from W. Schreiber in [6], see also M. Salerno in [6]). Within the trachea, the large bronchial tubes and also within the wide caverns of sick lung tissue one observes the free diffusion coefficient. In healthy lung tissue however, diffusion is apparently reduced by a factor 3 to 4 due to the small scale of its fractal structure. This fine structure also causes a kind of random field gradient through the susceptibility difference between lung tissue and the air spaces. This gradient scales with $B_{0}$ and leads to uncomfortably short coherence times at high field. Since hyperpolarization does not depend on $B_{0}$ one may take the chance to scale it down. An Orsay-Paris collaboration has explored low field ${ }^{3} \mathrm{He}$ MRI down to $0.1 \mathrm{~T}$ and observed the various benefits of enhanced coherence times [13].

\section{${ }^{129}$ Xe versus ${ }^{3} \mathrm{He}$}

For ${ }^{129} \mathrm{Xe}$ the signal situation is not as favourable as for ${ }^{3} \mathrm{He}$. The nuclear magnetic moment is three times smaller and the natural isotopic abundance is $29 \%$. Unless the very expensive enriched isotope is used, the magnetization drops by an order of magnitude as compared to ${ }^{3} \mathrm{He}$. That is a serious handicap for Xe but not a prohibitive one and may be easier to carry in a couple of years from now when MRI techniques may have progressed to even better signal to noise ratios. If in the long run a high clinical demand for this technique develops one will have to discuss the question of gas supply. At present, the ${ }^{3} \mathrm{He}$ market is fed only from the debris of tritium decay since ${ }^{3} \mathrm{He}$ abundance in natural helium is only 1.4 $\mathrm{ppm}$. A dedicated ${ }^{3} \mathrm{He}$ production will then be necessary which seems to be conceivable at reasonable terms. Xenon, on the other hand, is available from our atmosphere at any quantity desired. For economic reasons one would in any case recover the precious enriched ${ }^{129} \mathrm{Xe}$ as well as ${ }^{3} \mathrm{He}$ from the exhaled air; this is easily done. Satisfactory ${ }^{3} \mathrm{He}$ supply provided, hyperpolarized MRI of the lungs will probably stay with that isotope because it reaches by far the higher magnetization and is lacking any toxic side effect. Xenon also carries this latter handicap. Although its anaesthetic action is moderate for a single intake of breath, doctors prefer to avoid any toxicity associated with a treatment as far as possible.

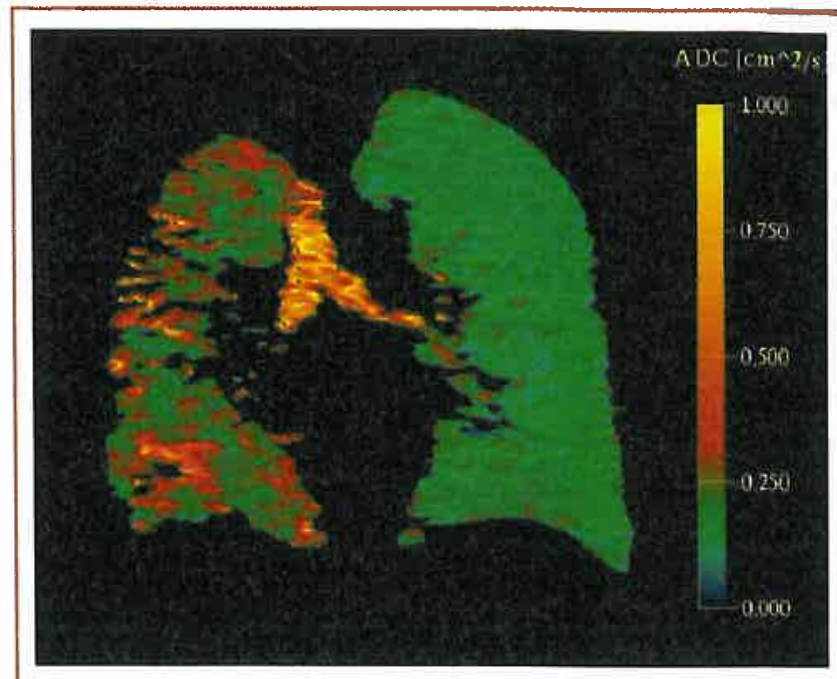

A Fig. 5: Mapping of the apparent diffusion coefficient of ${ }^{3} \mathrm{He}$ in the lungs of a patient suffering from fibrosis (left wing). It varies from $0.2 \mathrm{~cm}^{2} / \mathrm{s}$ in still functioning tissue to $0.8 \mathrm{~cm}^{2} / \mathrm{s}$ in wide caverns (see the colour code at the right). The black regions are not ventilated. The wing on the right has been transplanted and is well functioning.

Looking at ${ }^{129} \mathrm{Xe}$ only as a competitor to the use of ${ }^{3} \mathrm{He}$ would underestimate its role in MRI. Contrary to He it finds its chances explicitly through its strong surface interaction and solubility. This goes along with large characteristic chemical shifts of order $10^{-4}$ of its Larmor frequency, thus revealing its local host. Thus a group at Ann Arbor has identified ${ }^{129} \mathrm{Xe}$ in arterial and venous blood and also at different sites in the brain [14]. This opens, if not a widely applicable diagnostic tool, a very interesting research area, inaccessible to ${ }^{3} \mathrm{He}$ MRI. Therefore, hyperpolarized ${ }^{129} \mathrm{Xe}$ is also the preferred substance in a wide range of NMR studies at surfaces, in porous media etc. (see also K. Ruppert, S. Swanson, P. Blümler [6]).

\section{The medical case}

The images shown above in the context of MRI technology partly concern lungs already suffering from various diseases. Their effect on the images is apparently quite drastic and has never been shown before in this clarity and detail by any other diagnostic tool. The standard method for ventilation imaging is so far scintigraphy from inhaled radioactive gas, for instance ${ }^{81} \mathrm{Kr}$ or air loaded with ${ }^{99} \mathrm{Tc}$ aerosols. However, the in-plane resolution is poor, in the range of several $\mathrm{cm}$ only, and the depth is integrated up over the full lung. Hence, scintigraphy indicates an average regional ventilation. In order for a lung to function, it also needs to be perfused by blood. This has to be checked in addition by a separate perfusion scan obtained by some other radiological method. Oxygen-sensitive ${ }^{3} \mathrm{He}$ MRI can check this principal lung function in a single scan directly to the point. But what looks close to perfect already in the animal experiment (Fig. 4) needs some further development to yield precise results also for spontaneously breathing humans. Anyway, one is clearly en route from simple morphological to the more informative functional imaging at which doctors finally aim. In this sense also, the inhalation/exhalation movie is clearly functional imaging, because it answers the decisive question for any asthma or emphysema patient of how fast the gas is transported within the lungs. The static ventilation scan performed at the holding of breath yields superior spatial resolution in all three dimensions and 
clearly localizes any ventilation defect. Of great value is also the image of the diffusion coefficient since it measures the dimensions of the open structures down to the sub-millimetre scale of the alveolae. Hence, it discriminates between the finely structured healthy lung tissue with a high surface to volume ratio from inefficient blown-up cavities of a diseased lung (Fig. 5). Still, one has to remember that gas MRI has only access to ventilated air spaces. Neither non-ventilated open spaces nor the tissue itself contribute to the signal. Diseased tissue that may spread over the lung like cancer or fibrosis must still be diagnosed by other radiological means such as computer tomography (CT). The EU network PHIL [7] which has gathered European groups interested in ${ }^{3} \mathrm{He}$ MRI has confined its clinical aims to a systematic survey over about 200 emphysema patients in Denmark (Copenhagen), England (Sheffield) and Germany (Mainz). This large sample will form the base for establishing a reliable and differentiated ${ }^{3} \mathrm{He} \mathrm{MR}$ diagnosis of this, frequently encountered, serious disease. The survey is observing a fixed protocol of ${ }^{3} \mathrm{He}$ procedures comprising static and fast ventilation scans, a diffusion scan and in some cases an oxygen-sensitive scan. It is accompanied by complementary traditional diagnostic means on the same sample for comparison.

\section{Conclusion and outlook}

The physics of manipulating spins via OP and NMR seems to remain forever fresh due to a high rate of methodological innovations opening new fields of application in fundamental and applied science. Polarizing large quantities of noble gases to a high degree was a demand of particle and neutron physics in the case of ${ }^{3} \mathrm{He}$ and an aim of surface physics in the case of ${ }^{129} \mathrm{Xe}$. Then the potential of these polarized gases for MRI was discovered, in particular for imaging the air spaces in the lungs. This stimulated a new wave in radiology. Today, we count around 20 groups in the US and Europe who are actively pursuing research in this field. Approval of MR lung tomography for ${ }^{3} \mathrm{He}$ as a contrast agent is being sought on both continents and will probably soon pass the final Phase III. The further fate of the method, its clinical success, will then not lie predominantly in the hands of scientists anymore. However, they will have to assist industry and clinicians in the coming phase with extensive R\&D in order to optimize the methods and to realise the ultimate potential of the technique. The need for improved diagnostic tools is obviously there: Lung diseases are about number four in killing people and quite often after many years of painful suffering. Early diagnosis could help to prevent this fate.

\section{References}

[1] M. S. Albert et al., Nature 370, 199 (1994)

[2] P. J. Nacher and M. Leduc, J. Phys. (Paris) 46, 2057 (1985)

[3] P. L. Anthony et al., Phys. Rev. Lett. 71, 959 (1993)

[4] M. Meyerhoff et al., Phys. Rev. Lett. B 327, 201 (1994)

[5] R. Surkau et al., Nucl. Instr. \& Meth. A 384, 444 (1997)

[6] http://www.physik.uni-mainz.de/helion02

[7] http://www.phil.ens.fr

[8] W. Schreiber and R. Surkau, Phys. Blätter 55, 45 (1999)

[9] H.-U. Kauczor et al., Radiology 201, 564 (1996)

[10] http://imaging.med.virginia.edu/hyperpolarized/current.htm

[11] J. M. Wild et al., Magn. Res. Med. 49(6), 991 (2003)

[12] A. J. Deninger et al., Magn. Res. 141,207 (1999)

[13] E. Durand et al., Magn. Res, in Med. 47, 75 (2002)

[14] S. D. Swanson et al., Magn. Res. in Med. 38, 695 (1997)

\section{Czochralski's contribution:}

\section{0 years on}

Adam Gadomski, Institute of Mathematics and Physics, University of Technology and Agriculture, Bydgoszcz, Poland.

7 his article commemorates Jan Czochralski's contribution fifty I years after he passed away and was buried in his home town, Kcynia, roughly mid-way between Warsaw (Poland) and Berlin (Germany).

The seminal work published by Czochralski in Zeitschrift für physikalische Chemie, received for publication on August of $19^{\text {th }}$, 1916, but published two years later [1], was an example, rarely met in our days of conciseness and concreteness in presenting research results. On three pages the author reports on his new finding: that a method of pulling metallic monocrystals had been discovered, and had been applied for testing three metals of interest: $S n, P b$, and $\mathrm{Zn}$. This earned him much praise, and in consequence, he is still recognized as one of the fathers of todays semiconductor technology [2] although his method was thoroughly elaborated only for the above metals. It should be noted that Jan Czochralski invented his method during his stay and work in Berlin for the AEG (Allgemeine Elektrizitäts-Gesellschaft) company (Fig. 1) using the AEG lab facilities. He also benefitted from collaboration with a German physicist W. v. Mollendorff [3] and developed much of his knowledge on the crystallography of metals during this time.

In order to augment his activity in metal science and technology he moved from Berlin to Frankfurt on Main, where he worked intensively on metallurgical methods to obtain new alloys, as well as on metal based composites, leading to the production of bearings (metal $B$ ) [2], and on $A l$-based wires and contacts in electrotechnics. He also became interested in the durability of materials. However, with his German coworkers, his main focus was on the principles of the metallurgical processes, such as: the role of defects, additives, and specifically of dislocations, in efficiently yielding pure metals and alloys; stress-strain aspects; characteristics of recrystallisation; phase equilibria and transitions associated with their appearances, etc. It is also to be noted that during his time in Frankfurt he proposed in one of his papers "Radiotechnics in Service of Metal Science" in 1925 [4] a kind of radiomicroscope, serving to detect non-metallic inclusions in the outer layer (surface) of a metallic sample. This can in some way be

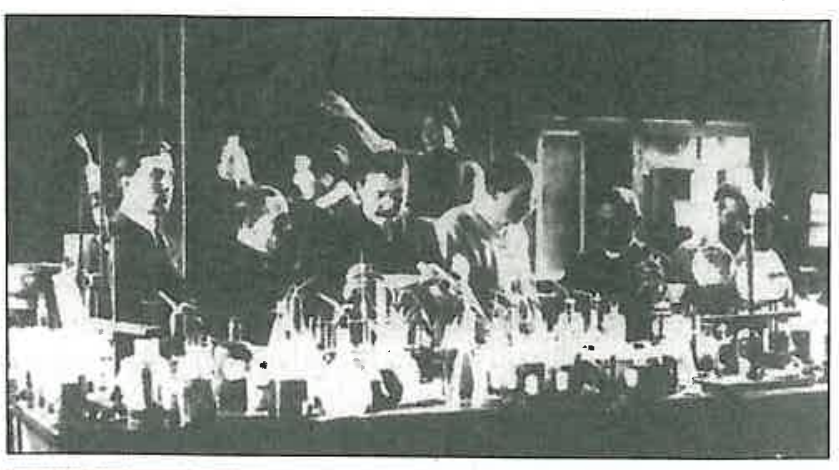

Aig. 1: Young Jan Czochralski (beginner), first from left, in an AEG lab in Berlin 1907 (courtesy of Z. Czochralska). 